\title{
Reachability and stabilization of scheduled steady-states for LPV single-input systems
}

\author{
Dario Corona $^{\mathrm{a}}$, Andrea Cristofaro ${ }^{\mathrm{b}}$, Damiano Rotondo ${ }^{\mathrm{c}, \mathrm{d}}$ \\ ${ }^{a}$ School of Science and Technology - Mathematics Division, University of Camerino, \\ Via Madonna delle Carceri 9, 62032 Camerino MC, Italy \\ ${ }^{b}$ Department of Technology Systems (ITS), University of Oslo, \\ Gunnar Randers vei 19, 2007 Kjeller, Norway \\ ${ }^{c}$ Institut de Robòtica i Informàtica Industrial, CSIC-UPC \\ Llorens i Artigas 4-6, 08028 Barcelona, Spain \\ ${ }^{d}$ Research Center for Supervision, Safety and Automatic Control (CS2AC) \\ Universitat Politècnica de Catalunya (UPC) \\ Rambla Sant Nebridi, s/n, 08022 Terrassa, Spain
}

\begin{abstract}
The aim of this work is characterizing the class of LPV systems that admit steady-state trajectories depending exclusively on the scheduling parameter. In particular, it will be shown that only certain parameter dependent steady-state profiles are admissible and can be reached by means of a suitable control input. Furthermore, the asymptotic stability and the stabilization of such steady-states is investigated using Lyapunov-based techniques. Extensive numerical simulations illustrate and corroborate the theoretical results.
\end{abstract}

Keywords: Linear-parameter varying systems, Asymptotic stability, Output regulation, Scheduled steady-states.

\section{Introduction}

The concept of steady-states, i.e. trajectories that are reached asymptotically or limit cycles, is well known in the framework of control systems. In particular, a simple yet powerful characterization is available for LTI systems [15], whereas formal definitions and properties have been derived for general classes of nonlinear control plants, e.g. input affine systems [9].

The aim of this paper is to develop similar concepts for the more challenging context of linear parameter varying (LPV) systems. LPV systems are a formalism which is often used in control engineering to model dynamics 
that strongly depend on some varying quantities, e.g. the operating point, so that linear-like analysis and design techniques can be applied $[1,26,19]$. For instance, by embedding the nonlinearities in the varying parameters using some exact transformation $[12,23]$, interpolating the Jacobian linearizations of the nonlinear system about several equilibrium points of interest [25], or performing data-driven identification [13], many nonlinear systems can be converted into a quasi-LPV form, where the word quasi refers to the dependence of the varying parameters on endogenous signals [14], in contrast with pure LPV systems, for which only dependence on exogenous signal is admitted.

Nowadays, the LPV framework is well-established as a possible alternative to more complex nonlinear analysis/design techniques, and has been validated by several experiments and high-fidelity simulations [8]. For instance, among many other applications, one can list: aviation [24, 29], robotic manipulators [10], automotive [7], motors [11], mobile robots [18] and biomedicine [4]. In addition to controller design, several theoretical problems of interest have been solved within the LPV framework, such as filtering [3, 16], fault detection [21, 20, 28] and fault tolerant control [22, 17].

In this work, we investigate the existence of scheduled steady-state trajectories (SSTs) for single-input LPV systems, i.e. limit trajectories depending on the instantaneous value of the scheduling parameters. In particular, this paper generalizes the results presented in [5], where the authors provided the characterization of a special class of LPV systems that admit constant SSTs. The results presented in this papers lay the theoretical foundations for performing more advanced tasks, such as the optimization of steady-state performance in reference tracking problems.

The paper is structured as follows. The basic notation is given at the end of the introduction. In Section 2, the formulation of the main problems is presented, together with the general underlying assumptions. In Section 3 , some necessary conditions for the solvability of the main problem are derived and discussed with respect to their geometric interpretation. Starting from such necessary conditions, Section 4 presents the main theorem of the paper, which provides sufficient conditions for the existence and uniqueness of SSTs, consisting in a geometric property and a differential relationship. In Section 5, the conditions for the stability of the scheduled steady-state trajectories are provided, together with the recipe for the synthesis of a stabilizing feedback loop. Finally, numerical simulations to corroborate the theoretical results are given in Section 6 . 


\section{Notation}

The set of real numbers is indicated by $\mathbb{R}$, while $\mathbb{R}^{+}$indicates the set of non-negative real numbers. For any scalar function $\phi: \mathbb{R}^{k} \rightarrow \mathbb{R}$, the gradient with respect to $z$ is denoted by $\nabla \phi(z) \in \mathbb{R}^{k}$. In particular, when $k=1$, one has $\nabla \phi(z)=\phi^{\prime}(z)$. If $\phi: \mathbb{R}^{+} \rightarrow \mathbb{R}^{k}$ is a function of time, its derivative is denoted by $\dot{\phi}(t) \in \mathbb{R}^{k}$. For any vector function $f: \mathbb{R}^{k} \rightarrow \mathbb{R}^{s}$, we denote by $\mathbf{D} f(z) \in \mathbb{R}^{s \times k}$ the Jacobian matrix of $f$ with respect to $z$

$$
\mathbf{D} f(z)=\left[\begin{array}{ccc}
\frac{\partial f_{1}}{\partial z_{1}}(z) & \ldots & \frac{\partial f_{1}}{\partial z_{k}}(z) \\
\vdots & \ddots & \vdots \\
\frac{\partial f_{s}}{\partial z_{1}}(z) & \ldots & \frac{\partial f_{s}}{\partial z_{k}}(z)
\end{array}\right] .
$$

Accordingly, if $\phi: \mathbb{R}^{+} \rightarrow \mathbb{R}^{k}$ and $f: \mathbb{R}^{k} \rightarrow \mathbb{R}^{s}$, the composition of functions satisfies the chain rule

$$
\frac{d(f(\phi(t))}{d t}=\mathbf{D} f(\phi(t)) \dot{\phi}(t) \in \mathbb{R}^{s}
$$

Given a real matrix $M \in \mathbb{R}^{k \times s}, M^{\dagger} \in \mathbb{R}^{s \times k}$ denotes its Moore-Penrose inverse. For a square matrix $M \in \mathbb{R}^{k \times k},|M| \in \mathbb{R}$ denotes its determinant. Given two vectors $v, w \in \mathbb{R}^{k},\langle v, w\rangle \in \mathbb{R}$ indicates the usual dot product, while the notation $v \| w$ indicates that the vectors are parallel.

\section{Problem setting}

Let $\Sigma(\theta, \dot{\theta})=(A(\theta, \dot{\theta}), b(\theta, \dot{\theta}))$ be the continuous-time LPV system

$$
\dot{x}(t)=A(\theta, \dot{\theta}) x(t)+b(\theta, \dot{\theta}) u(t)
$$

with $A(\theta, \dot{\theta}) \in \mathbb{R}^{n \times n}, b(\theta, \dot{\theta}) \in \mathbb{R}^{n \times 1}$. The input $u(t)$ is assumed to belong to the set of piecewise continuous and bounded functions $\mathcal{U}$. The vector $\theta \in \mathbb{R}^{z}$, $z \geq 1$, represents the scheduled time-varying parameters, and is allowed to assume values in a closed and bounded set $\Theta \subset \mathbb{R}^{z}$. Furthermore, we assume the existence of the time derivative

$$
\dot{\theta}=\left[\begin{array}{lll}
\frac{d \theta_{1}}{d t} & \cdots & \frac{d \theta_{n}}{d t}
\end{array}\right]^{T} \in \mathbb{R}^{z}
$$

almost everywhere. Let us point out that, if $t=\bar{t}$ is a non-differentiability point for $\theta(t)$, then both left and right derivative $\dot{\theta}\left(\bar{t}^{-}\right)$and $\dot{\theta}\left(\bar{t}^{+}\right)$are assumed to exist and to be finite. 
Throughout this work, it will be suitable to see $\theta(\cdot)$ as functions of time, and the set of admissible scheduled parameters trajectories is denoted by

$$
\mathcal{H}:=\left\{\theta: \mathbb{R}^{+} \rightarrow \Theta, \quad \dot{\theta}(t) \in \mathbb{R}^{z} \forall \text { a.e. } t \in \mathbb{R}^{+}\right\}
$$

Our main objective is to investigate whether the LPV system admits steadystates that can be expressed as pure functions of the scheduled parameters. In this regard, let us introduce the following definition.

Definition 1. A function $v: \Theta \rightarrow \mathbb{R}^{n}$ is said to be a reachable scheduled profile (RSP) if, given $t_{0} \in \mathbb{R}^{+}$such that $x\left(t_{0}\right)=v\left(\tilde{\theta}\left(t_{0}\right)\right)$, there exists a control input $u \in \mathcal{U}$ such that, for any given $\tilde{\theta} \in \mathcal{H}$, the corresponding state response satisfies

$$
x(t)=v(\tilde{\theta}(t)) \quad \forall t \geq t_{0} .
$$

The set of all the RSPs is denoted by $\mathcal{V}$.

An element $v \in \mathcal{V}$ describes a reachable SST of the system, irrespectively of the particular time realization $\tilde{\theta} \in \mathcal{H}$. Observe that $\mathcal{V} \neq \emptyset$ for each LPV system: in fact the zero function $v: \Theta \rightarrow \mathbb{R}^{n}, v(\theta) \equiv 0$ is always in $\mathcal{V}$, and can be regarded as the trivial RSP. The set of all possible solutions different from the trivial one is denoted by $\overline{\mathcal{V}}=\mathcal{V} \backslash\{0\}$. With a slight abuse of notation, we may sometimes refer to $v \in \mathcal{V}$ as a function of time through the composition with a particular $\tilde{\theta} \in \mathcal{H}$, and this will become clear from the context. In this regard, by the chain rule (1), the derivative of $v$ with respect to time is given by

$$
\frac{d v}{d t}(t)=\mathbf{D} v(\theta(t)) \dot{\theta}(t) \in \mathbb{R}^{n} .
$$

From Definition 1 , the feasibility of $v \in \overline{\mathcal{V}}$ is related to the existence of a proper input function $u(\theta, \dot{\theta})$ such that

$$
\mathbf{D} v(\theta) \dot{\theta}=A(\theta, \dot{\theta}) v(\theta)+b(\theta, \dot{\theta}) u(\theta, \dot{\theta}) \quad \forall \theta \in \Theta, \forall \dot{\theta} \in \mathbb{R}^{z} .
$$

Moreover, if such input function exists, it is also unique modulo the condition $b(\theta, \dot{\theta}) \neq 0 \forall(\theta, \dot{\theta}) \in \Theta \times \mathbb{R}^{z}$. In fact, if two inputs $u_{1}, u_{2} \in \mathcal{U}$ satisfy (4) for the same $v \in \mathcal{V}$, then

$$
b(\theta, \dot{\theta})\left(u_{1}(\theta, \dot{\theta})-u_{2}(\theta, \dot{\theta})\right)=0 \quad \forall(\theta, \dot{\theta}) \in \Theta \times \mathbb{R}^{z}
$$

and thus, as long as $b(\theta, \dot{\theta})$ is a non-zero vector, one has $u_{1}=u_{2} \forall \theta \in \Theta, \forall \dot{\theta} \in$ $\mathbb{R}^{z}$. Given $v \in \mathcal{V}$, the corresponding input provided by condition (4) will be 
denoted by $u_{v} \in \mathcal{U}$. The main objective of the paper can be then formally stated as follows.

\section{Problem 1:}

(i) find sufficient conditions on the LPV state-space matrices $A(\theta, \dot{\theta})$ and $b(\theta, \dot{\theta})$ in order to have $\overline{\mathcal{V}} \neq \emptyset ;$

(ii) determine the analytical expressions of $v \in \overline{\mathcal{V}}$, along with their corresponding input functions $u_{v}$.

Remark 1. It is worth noting that (4) prescribes a global condition on $v$, i.e. a partial differential equation that has to be satisfied for each couple $(\theta, \dot{\theta})$. Indeed, for each $\tilde{\theta} \in \mathcal{H}, v(\tilde{\theta})$ is a steady state trajectory driven by a proper input function. Accordingly, $v$ implicitly defines a mapping $u_{v}: \mathcal{H} \rightarrow \mathcal{U}$ where $u_{v}(\tilde{\theta})$ is the input that solves (4) for the function $v \in \overline{\mathcal{V}}$.

Remark 2. The problem stated in this section, and addressed in the rest of the paper, deals with state trajectories. Similar results may be obtained for output trajectories, augmenting the LPV system (2) with an output equation

$$
y(t)=C(\theta, \dot{\theta}) x(t)+d(\theta, \dot{\theta}) u(t) .
$$

However, the mathematical development of this case goes beyond the scope of this paper, and as such, is omitted.

\section{Characterization of RSP}

In this section, some preliminary, and necessary, conditions on the RPSs and the structure of $\mathcal{V}$ will be established, whereas the problem of existence will be formally addressed later on.

As a starting point, the next result shows that the admissible values of $v(\theta)$ are constrained onto a linear subspace of $\mathbb{R}^{n}$.

Theorem 1. A necessary condition for having a nontrivial $\overline{\mathcal{V}} \neq \emptyset$ is

$$
\operatorname{rank}\{A(\theta, 0)\}=\operatorname{rank}\{[A(\theta, 0) \mid b(\theta, 0)]\} .
$$

If this condition holds, then for any admissible $v \in \mathcal{V}$ there exist two functions $\rho: \Theta \rightarrow \mathbb{R}$ and $w: \Theta \rightarrow \mathbb{R}^{n}$ such that

$$
v(\theta)=A^{\dagger}(\theta, 0) b(\theta, 0) \rho(\theta)+\left[I-A^{\dagger}(\theta, 0) A(\theta, 0)\right] w(\theta) .
$$


Proof. If $v \in \mathcal{V}$, then $v(\tilde{\theta})$ is a steady-state trajectory $\forall \tilde{\theta} \in \mathcal{H}$ under the input $u_{v}(\tilde{\theta}) \in \mathcal{U}$. In particular, it must be a steady-state trajectory for every constant function $\tilde{\theta}(t) \equiv \theta$. In this case, condition (4) becomes

$$
A(\theta, 0) v(\theta)=-b(\theta, 0) u_{v}(\theta, 0) .
$$

Since $u_{v}(\theta, 0)$ is a scalar, all solutions of the latter equation other than the trivial one must be parallel to a vector $\xi \in \mathbb{R}^{n}$ such that

$$
A(\theta, 0) \xi=b(\theta, 0) \text {. }
$$

On the other hand, such $\xi$ exists if and only if (5) holds, which is, accordingly, a necessary condition for the existence of $v \in \overline{\mathcal{V}}$. In this case, all the possible solutions of $A(\theta, 0) \xi=b(\theta, 0)$ are given by

$$
\xi=A^{\dagger}(\theta, 0) b(\theta, 0)+\left[I-A^{\dagger}(\theta, 0) A(\theta, 0)\right] \tilde{w},
$$

with $\tilde{w} \in \mathbb{R}^{n}$. From the Moore-Penrose pseudo-inverse definition, one has

$$
\left\langle A^{\dagger}(\theta, 0) b(\theta, 0),\left[I-A^{\dagger}(\theta, 0) A(\theta, 0)\right] w\right\rangle=0 \quad \forall w \in \mathbb{R}^{n} .
$$

Consequently, since $\tilde{w}$ is an arbitrary vector and $v(\theta)$ must be, for all $\theta \in \Theta$, a vector parallel to any $\xi$ given by (8), there exist necessarily $\rho: \Theta \rightarrow \mathbb{R}$ and $w: \Theta \rightarrow \mathbb{R}^{n}$ such that (6) holds.

An immediate consequence of this theorem is that, if $v \in \mathcal{V}$, then $\forall \theta \in \Theta$ $v(\theta)$ must lie in a linear subspace of $\mathbb{R}^{n}$ which depends only on $A(\theta, 0)$ and $b(\theta, 0)$. In fact, from $(6), v(\theta)$ belongs to the direct sum of two linear and orthogonal spaces given by $A^{\dagger}(\theta, 0) b(\theta, 0)$ and $\operatorname{ker}\{A(\theta, 0)\}$. As a consequence, the dimension of such linear subspace is $1+n-\operatorname{rank}\{A(\theta, 0)\}$.

Corollary. If $A(\theta, 0)$ is an invertible matrix for each $\theta \in \Theta$, then all the admissible $v \in \mathcal{V}$ are given by

$$
v(\theta)=A(\theta, 0)^{-1} b(\theta, 0) \rho(\theta),
$$

with $\rho: \Theta \rightarrow \mathbb{R}$.

Remark 3. The previous corollary is a generalization of the result provided in [5, Theorem 1], which established the existence of constant steady state trajectories for single input LPV-systems of the following kind

$$
\dot{x}=A(\theta) x+b(\theta) u \text {. }
$$

In such case, the function $v(\theta)$ given by (9) turns out to be a constant, independent of $\theta$. 


\section{Existence of RSP}

In general, providing sufficient conditions for the existence of RSPs is a difficult task, since the analysis of both $\rho$ and $w$ in (6) is required. Throughout this section, in order to derive some closed-form results, the matrix $A(\theta, 0)$ will be assumed to be invertible, so that only the function $\rho: \Theta \rightarrow \mathbb{R}$ has to be taken into account. Based on this standing assumption, an analytical form of the admissible $v \in \mathcal{V}$ together with the corresponding inputs $u_{v} \in \mathcal{U}$ is obtained, thus addressing the main problem of this work. Let us point out that such characterization hinges on the solution to a system of first-order partial differential equations.

Since $A(\theta, 0)$ is an invertible matrix, defining the function $k: \Theta \rightarrow \mathbb{R}^{n}$ as

$$
k(\theta)=A(\theta, 0)^{-1} b(\theta, 0) \in \mathbb{R}^{n},
$$

it follows from Theorem 1 that one can restrict the analysis on candidates $v \in \mathcal{V}$ of the particular form

$$
v(\theta)=k(\theta) \rho(\theta)
$$

Let us state some preliminary facts. For a RSP given by (11), equation (4) reads as

$$
[\mathbf{D} k(\theta) \rho(\theta)+k(\theta) \nabla \rho(\theta)] \dot{\theta}=A(\theta, \dot{\theta}) k(\theta) \rho(\theta)+b(\theta, \dot{\theta}) u_{v} .
$$

Introducing

$$
\begin{gathered}
g(\theta, \dot{\theta})=\mathbf{D} k(\theta) \dot{\theta}-A(\theta, \dot{\theta}) k(\theta) \in \mathbb{R}^{n} \\
F(\theta, \dot{\theta})=k(\theta) \dot{\theta}^{T} \in \mathbb{R}^{n \times z},
\end{gathered}
$$

and observing that $\nabla \rho(\theta) \dot{\theta}=\dot{\theta}^{T} \nabla^{T} \rho(\theta) \in \mathbb{R}$, identity (12) can be resorted as

$$
g(\theta, \dot{\theta}) \rho(\theta)+F(\theta, \dot{\theta}) \nabla^{T} \rho(\theta)=b(\theta, \dot{\theta}) u_{v},
$$

which constitutes a system of partial differential equations. Let us consider the singular values decomposition

$$
F(\theta, \dot{\theta})=S(\theta, \dot{\theta}) Z(\theta, \dot{\theta}) V(\theta, \dot{\theta})
$$

where $S(\theta, \dot{\theta}) \in \mathbb{R}^{n \times n}$ and $V(\theta, \dot{\theta}) \in \mathbb{R}^{z \times z}$ are two unitary matrices and $Z(\theta, \dot{\theta}) \in \mathbb{R}^{n \times z}$ is a rectangular diagonal matrix containing the singular values of $F(\theta, \dot{\theta})$. Let us notice that, since $k(\theta)$ and $\dot{\theta}$ are vectors, then 
$\operatorname{rank}\{F(\theta, \dot{\theta})\}=1$ and $Z(\theta, \dot{\theta})$ has only one non-zero entry. In particular, without loss of generality, $Z(\theta, \dot{\theta})$ can be supposed of the following form

$$
Z(\theta, \dot{\theta})=\left[\begin{array}{c|c}
\xi(\theta, \dot{\theta}) & \mathbf{0} \\
\hline \mathbf{0} & \mathbf{0}
\end{array}\right], \quad \xi(\theta, \dot{\theta}) \in \mathbb{R}^{+} .
$$

Using (16), equation (15) can be rewritten as

$$
S(\theta, \dot{\theta})^{-1} g(\theta, \dot{\theta}) \rho(\theta)+Z(\theta, \dot{\theta}) V(\theta, \dot{\theta}) \nabla^{T} \rho(\theta)=S(\theta, \dot{\theta})^{-1} b(\theta, \dot{\theta}) u .
$$

Now, let $S_{1}(\theta, \dot{\theta}) \in \mathbb{R}^{1 \times n}$ and $S_{2}(\theta, \dot{\theta}) \in \mathbb{R}^{(n-1) \times n}$ be selected such that the following decomposition holds

$$
S^{-1}(\theta, \dot{\theta})=\left[\frac{S_{1}(\theta, \dot{\theta})}{S_{2}(\theta, \dot{\theta})}\right]
$$

Accordingly, the system of partial differential equations (18) is equivalent to

$$
\left\{\begin{array}{l}
S_{1}(\theta, \dot{\theta}) g(\theta, \dot{\theta}) \rho(\theta)+\xi(\theta, \dot{\theta}) V(\theta, \dot{\theta}) \nabla^{T} \rho(\theta)=S_{1}(\theta, \dot{\theta}) b(\theta, \dot{\theta}) u_{v} \\
S_{2}(\theta, \dot{\theta}) g(\theta, \dot{\theta}) \rho(\theta)=S_{2}(\theta, \dot{\theta}) b(\theta, \dot{\theta}) u_{v}
\end{array}\right.
$$

It is worth noting that all the previous conditions are both necessary and sufficient for the existence of $v \in \mathcal{V}$. Indeed, the system (20-21) is equivalent to (4).

Theorem 2. Let $A(\theta, 0)$ be an invertible matrix for any $\theta \in \Theta$. Then the candidate $R S P v(\theta)=k(\theta) \rho(\theta)$ is in $\overline{\mathcal{V}}$ if and only if:

(i) $\exists \lambda(\theta, \dot{\theta}) \in \mathbb{R} \backslash\{0\}$ such that

$$
S_{2}(\theta, \dot{\theta}) g(\theta, \dot{\theta})=\lambda(\theta, \dot{\theta}) S_{2}(\theta, \dot{\theta}) b(\theta, \dot{\theta})
$$

(ii) setting

$$
\alpha(\theta, \dot{\theta})=S_{1}(\theta, \dot{\theta})[\lambda(\theta, \dot{\theta}) b(\theta, \dot{\theta})-g(\theta, \dot{\theta})] \in \mathbb{R}
$$

the partial differential equation

$$
\xi(\theta, \dot{\theta}) \sum_{i=1}^{z} V_{1 i}(\theta, \dot{\theta}) \frac{\partial \rho}{\partial \theta_{i}}(\theta)=\alpha(\theta, \dot{\theta}) \rho(\theta)
$$

admits a non-trivial solution $\rho(\theta)$ that does not depend on $\dot{\theta}$. 
If the two latter conditions are fulfilled, the corresponding input function is then given by

$$
u_{v}(\theta, \dot{\theta})=\lambda(\theta, \dot{\theta}) \rho(\theta) .
$$

Proof. A necessary condition for the existence of $u_{v} \in \mathcal{U}$ and $\rho: \Theta \rightarrow \mathbb{R}$ satisfying (21) is

$$
S_{2}(\theta, \dot{\theta}) g(\theta, \dot{\theta}) \| S_{2}(\theta, \dot{\theta}) b(\theta, \dot{\theta})
$$

or, equivalently, the existence of $\lambda(\theta, \dot{\theta})$ such that identity (22) is fulfilled. In that case, $u_{v}(\theta, \dot{\theta})=\lambda(\theta, \dot{\theta}) \rho(\theta)$ and (20) expands as follows

$$
S_{1}(\theta, \dot{\theta}) g(\theta, \dot{\theta}) \rho(\theta)+\xi(\theta, \dot{\theta}) V(\theta, \dot{\theta}) \nabla^{T} \rho(\theta)=\lambda(\theta, \dot{\theta}) S_{1}(\theta, \dot{\theta}) b(\theta, \dot{\theta}) \rho(\theta) .
$$

Using $\alpha(\theta, \dot{\theta})$ as defined in (23), the latter is

$$
\xi(\theta, \dot{\theta}) V(\theta, \dot{\theta}) \nabla^{T} \rho(\theta)=\alpha(\theta, \dot{\theta}) \rho(\theta),
$$

which is in turn equivalent to (24). Finally, if there exists a solution $\rho(\theta)$ to (20) which does not depend on $\dot{\theta}$, such solution makes $v(\theta)$ in (11) a RSP under the input $u_{v}$ given by $(25)$.

Theorem 2 states two different conditions for the existence of a RSP. Identity (21) is a geometric structural condition and depends only on the plant matrices, while $(20)$ is a differential condition on $\rho(\theta)$. In particular, since (20) leads to a linear first order PDE (24), the next result follows.

Corollary. The set $\mathcal{V}$ is a vector space.

Proof. Let $v_{1}=k(\theta) \rho_{1}(\theta), v_{2}=k(\theta) \rho_{2}(\theta)$ be two independent functions in $\mathcal{V}$. Then $\rho_{1}, \rho_{2}$ solve $(24)$, along with any combination $\rho_{3}=\gamma_{1} \rho_{1}+$ $\gamma_{2} \rho_{2} \forall \gamma_{1}, \gamma_{2} \in \mathbb{R}$. As a consequence

$$
v(\theta)=\gamma_{1} v_{1}(\theta)+\gamma_{2} v_{2}(\theta)=k(\theta)\left[\gamma_{1} \rho_{1}(\theta)+\gamma_{2} \rho_{2}(\theta)\right]=k(\theta) \rho_{3}(\theta) \in \mathcal{V}
$$

for any $\gamma_{1}, \gamma_{2} \in \mathbb{R}$ and its corresponding input is given by the linear relationship $u_{v}(\theta, \dot{\theta})=\gamma_{1} u_{v_{1}}(\theta, \dot{\theta})+\gamma_{2} u_{v_{2}}(\theta, \dot{\theta})$.

Let us stress that, as (24) is a partial differential equation defined on a space embedded in $\mathbb{R}^{z}$, in order to achieve uniqueness of solution it is in general necessary to assign the value of the solution on a $(z-1)$-dimensional manifold. As a consequence, a $v \in \mathcal{V}$ which solves

$$
\left\{\begin{array}{l}
\mathbf{D} v \dot{\theta}=A(\theta, \dot{\theta}) v+b(\theta, \dot{\theta}) u_{v}(\theta, \dot{\theta}) \\
v(\bar{\theta})=v_{0}
\end{array}\right.
$$


can not be expected to be unique for $z>1$. A deeper analysis of possible solutions of (24), together with their properties, goes beyond the purpose of this work. Nevertheless, a special case where uniqueness is attained is discussed next.

\subsection{Two dimensional case}

Let us analyze the case of a two-dimensional system with a scalar scheduling parameter, i.e. $n=2$ and $z=1$. In such a case, a simpler condition to guarantee the existence of RSPs can be established. Towards this goal, let us observe that (15) becomes

$$
\left\{\begin{array}{l}
g_{1}(\theta, \dot{\theta}) \rho(\theta)+f_{1}(\theta, \dot{\theta}) \rho^{\prime}(\theta)=b_{1}(\theta, \dot{\theta}) u \\
g_{2}(\theta, \dot{\theta}) \rho(\theta)+f_{2}(\theta, \dot{\theta}) \rho^{\prime}(\theta)=b_{2}(\theta, \dot{\theta}) u
\end{array}\right.
$$

with $f(\theta, \dot{\theta})=k(\theta) \dot{\theta} \in \mathbb{R}^{2}$. Assuming for the sake of simplicity $b(\theta, \dot{\theta}) \neq 0$ and solving for $u_{v}$, the following differential equation is obtained

$$
\left(b_{1}(\theta, \dot{\theta}) g_{2}(\theta, \dot{\theta})-b_{2}(\theta, \dot{\theta}) g_{1}(\theta, \dot{\theta})\right) \rho(\theta)=\left(b_{2}(\theta, \dot{\theta}) f_{1}(\theta, \dot{\theta})-b_{1}(\theta, \dot{\theta}) f_{2}(\theta, \dot{\theta})\right) \rho^{\prime}(\theta) \text {. }
$$

A solution $\rho(\theta) \neq 0$ with the desired properties can be found if

$$
h(\theta):=-\frac{b_{1}(\theta, \dot{\theta}) g_{2}(\theta, \dot{\theta})-b_{2}(\theta, \dot{\theta}) g_{1}(\theta, \dot{\theta})}{b_{1}(\theta, \dot{\theta}) f_{2}(\theta, \dot{\theta})-b_{2}(\theta, \dot{\theta}) f_{1}(\theta, \dot{\theta})}
$$

does not depend on $\dot{\theta}$. In that case one can set

$$
\rho(\theta)=\lambda e^{\int h(\theta) d \theta} \quad \lambda \in \mathbb{R},
$$

with corresponding input given by

$$
u(\theta, \dot{\theta})=\frac{g_{1}(\theta, \dot{\theta}) \rho(\theta)+f_{1}(\theta, \dot{\theta}) \rho^{\prime}(\theta)}{b_{1}(\theta, \dot{\theta})} .
$$

In this lower dimensional case, the condition (26) is sufficient to guarantee the existence of RSPs. In fact, the geometric condition given by (21) is trivially satisfied by a two-dimensional plant. Moreover, if (26) holds, (27) and (28) give the analytic expressions of the RSP and the control input respectively.

Let us notice that, for instance, condition (26) is fulfilled by any system of the form

$$
\dot{x}=(\bar{A}(\theta)+\dot{\theta} \hat{A}(\theta)) x+\hat{b}(\theta) \beta(\dot{\theta}) u,
$$


i.e., with

$$
A(\theta, \dot{\theta})=\bar{A}(\theta)+\dot{\theta} \hat{A}(\theta) \quad|\bar{A}(\theta)| \neq 0 \forall \theta
$$

and

$$
b(\theta, \dot{\theta})=\hat{b}(\theta) \beta(\dot{\theta}) \quad \beta(\dot{\theta}) \neq 0 \forall \dot{\theta} .
$$

In this case, Eq. (13) becomes

$$
g(\theta, \dot{\theta})=\mathbf{D} k(\theta) \dot{\theta}-b(\theta, 0)-\dot{\theta} \hat{A}(\theta) k(\theta),
$$

and the numerator of (26) is given by

$$
\begin{gathered}
\beta(\dot{\theta})\left[\dot{\theta} \hat{b}_{1}(\theta) \mathbf{D}_{2} k(\theta)-\hat{b}_{1}(\theta) \hat{b}_{2}(\theta) \beta(0)-\dot{\theta} \hat{b}_{1}(\theta) \hat{A}_{2}(\theta) k(\theta)-\right. \\
\left.\dot{\theta} \hat{b}_{2}(\theta) \mathbf{D}_{1} k(\theta)+\hat{b}_{1}(\theta) \hat{b}_{2}(\theta) \beta(0)+\dot{\theta} \hat{b}_{2}(\theta) \hat{A}_{1}(\theta) k(\theta)\right]= \\
\beta(\dot{\theta}) \dot{\theta}\left[\hat{b}_{1}(\theta) \mathbf{D}_{2} k(\theta)-\hat{b}_{1}(\theta) \hat{A}_{2}(\theta) k(\theta)-\hat{b}_{2}(\theta) \mathbf{D}_{1} k(\theta)+\hat{b}_{2}(\theta) \hat{A}_{1}(\theta) k(\theta)\right],
\end{gathered}
$$

while the denominator is

$$
\beta(\dot{\theta}) \dot{\theta}\left[\hat{b}_{1}(\theta) k_{2}(\theta)-\hat{b}_{2}(\theta) k_{1}(\theta)\right] .
$$

As a consequence, up to basic algebraic computations, it results

$$
h(\theta)=-\frac{\hat{b}_{1}(\theta) \mathbf{D}_{2} k(\theta)-\hat{b}_{1}(\theta) \hat{A}_{2}(\theta) k(\theta)-\hat{b}_{2}(\theta) \mathbf{D}_{1} k(\theta)+\hat{b}_{2}(\theta) \hat{A}_{1}(\theta) k(\theta)}{\hat{b}_{1}(\theta) k_{2}(\theta)-\hat{b}_{2}(\theta) k_{1}(\theta)},
$$

which is independent of $\dot{\theta}$, as required.

\section{Stability and stabilization of steady-states}

Theorem 2 provides sufficient conditions for the existence of reachable scheduled profiles. Hereafter, the asymptotic stability of the corresponding state trajectories is derived from the asymptotic stability of the origin with $u(t)=0$. Let us recall that the origin is an asymptotically stable equilibrium state for bounded-rate variations of the varying parameter $\theta(t)$ in $\Theta$, with $\dot{\theta} \in \dot{\Theta} \subseteq \mathbb{R}^{z}$ and $u(t)=0$, if there exists a parameter-dependent Lyapunov function of the form $[6]$

$$
V(x(t), \theta(t))=x(t)^{T} P(x(t), \theta(t)) x(t)
$$

with $P(x(t), \theta(t))=P(x(t), \theta(t))^{T}>0$ and

$$
\dot{V}(x(t), \theta(t)) \forall(x, \theta, \dot{\theta}) \in \mathbb{R} \backslash\{0\} \times \Theta \times \dot{\Theta} .
$$

If $\dot{\Theta}=\mathbb{R}^{z}$, then stability for arbitrary time variations of $\theta(t)$ is obtained. Notice that the Lyapunov condition $\dot{V}(x(t), \theta(t))<0$ for $(33)$ reads as:

$$
A(\theta, \dot{\theta})^{T} P(x, \theta)+P(x, \theta) A(\theta, \dot{\theta})+\dot{P}(x, \theta)<0
$$


Theorem 3. Let $v \in \overline{\mathcal{V}}$ be an arbitrary RSP. If the origin is an asymptotically stable equilibrium state for the LPV system (2) in the uncontrolled case $u(t)=0$, then the $R S P v(\theta)$ is asymptotically stable for the system subject to the input $u_{v}(\theta, \dot{\theta})$.

Proof. Consider the Lyapunov function:

$$
\Psi(x, \theta)=(x-v(\theta))^{T} P(x, \theta)(x-v(\theta))
$$

whose derivative is given by:

$$
\begin{aligned}
\dot{\Psi}(x, u, \theta, \dot{\theta})= & 2(x-v(\theta))^{T} P(x, \theta)(A(\theta, \dot{\theta}) x+b(\theta, \dot{\theta}) u-\mathbf{D} v(\theta) \dot{\theta})+ \\
& (x-v(\theta))^{T} \dot{P}(x, \theta)(x-v(\theta))
\end{aligned}
$$

From the definition of $u_{v} \in \mathcal{U}$, one has

$$
A(\theta, \dot{\theta}) v(\theta)=\mathbf{D} v(\theta) \dot{\theta}-b(\theta, \dot{\theta}) u_{v}(\theta, \dot{\theta})
$$

and thus (35) can be rewritten as

$$
\begin{aligned}
& \dot{\Psi}(x, u, \theta, \dot{\theta})=(x-v(\theta))^{T}[2 P(x, \theta) A(\theta, \dot{\theta})+\dot{P}(x, \theta)](x-v(\theta)) \\
& \quad=(x-v(\theta))^{T}\left[A(\theta, \dot{\theta})^{T} P(x, \theta)+P(x, \theta) A(\theta, \dot{\theta})+\dot{P}(x, \theta)\right](x-v(\theta))
\end{aligned}
$$

The negative definiteness of $\dot{\Psi}(x, u, \theta, \dot{\theta})$ is then inherited from the uncontrolled condition (34), and therefore it follows that the parameter-varying steady-state trajectory $x_{\infty}(\theta)=v(\theta)$ is asymptotically stable under the input $u_{v}(\theta, \dot{\theta})$.

The assumption of the asymptotic stability of the origin can be weakened, assuming the existence of a controller gain $k(\theta, \dot{\theta})$ such that the closed-loop system

$$
\dot{x}=\left(A(\theta, \dot{\theta})+b(\theta, \dot{\theta}) k^{T}(\theta, \dot{\theta})\right) x+b(\theta, \dot{\theta}) u
$$

results to be asymptotically stable. In fact, using the input

$$
u=u_{v}(\theta, \dot{\theta})+u_{f}(x, \theta)=u_{v}(\theta, \dot{\theta})+k(\theta, \dot{\theta})^{T}(x-v(\theta))
$$

in (2) leads to the stable system

$$
\dot{x}=\left(A(\theta, \dot{\theta})+b(\theta, \dot{\theta}) k^{T}(\theta, \dot{\theta})\right) x+b(\theta, \dot{\theta})\left(u_{v}(\theta, \dot{\theta})-k(\theta, \dot{\theta})^{T} v(\theta)\right),
$$

where the input $u_{v}(\theta, \dot{\theta})-k(\theta, \dot{\theta})^{T} v(\theta)$ trivially satisfies (4) for any $v \in \mathcal{V}$. 


\section{Numerical Examples}

\section{1. $A(\theta, 0)$ invertible}

The first proposed example illustrates the results given in Section 4.1. Let us consider an LPV plant with

$$
A(\theta, \dot{\theta})=\bar{A}(\theta)+\dot{\theta} \hat{A}(\theta)=\left[\begin{array}{cc}
-1 & \theta \\
0 & -3
\end{array}\right]+\dot{\theta}\left[\begin{array}{cc}
-3 & 1 \\
2 & -4
\end{array}\right],
$$

and

$$
b(\theta, \dot{\theta})=\hat{b}(\theta) \beta(\dot{\theta})=\left[\begin{array}{l}
1 \\
2
\end{array}\right]=b .
$$

Accordingly, we can define

$$
\begin{aligned}
& k(\theta)=\bar{A}^{-1}(\theta) b=\frac{1}{3}\left[\begin{array}{c}
-3-2 \theta \\
-2
\end{array}\right], \\
& g(\theta, \dot{\theta})=-\left[\begin{array}{l}
1 \\
2
\end{array}\right]-\dot{\theta}\left[\begin{array}{l}
3+2 \theta \\
\frac{2}{3}-\frac{4}{3} \theta
\end{array}\right], \\
& f(\theta, \dot{\theta})=k(\theta) \dot{\theta},
\end{aligned}
$$

and, after some algebraic manipulations, condition (26) becomes

$$
\rho^{\prime}(\theta) / \rho(\theta)=h(\theta)=-4,
$$

which provides the solution $\rho(\theta)=\lambda e^{-4 \theta}, \quad \lambda \in \mathbb{R}$. In this case, the RSP and the corresponding input turns out to be respectively

$$
\begin{aligned}
v(\theta) & =-\frac{1}{3} \lambda\left[\begin{array}{c}
3+2 \theta \\
2
\end{array}\right] e^{-4 \theta} \\
u_{v}(\theta, \dot{\theta}) & =\lambda e^{-4 \theta}\left(\dot{\theta}+\frac{2}{3} \theta \dot{\theta}-1\right) .
\end{aligned}
$$

The same results may be obtained using the singular-value decomposition method based on equations (13)-(25). The singular value decomposition of $f(\theta, \dot{\theta})$ is

$$
f(\theta, \dot{\theta})=S(\theta, \dot{\theta}) Z(\theta, \dot{\theta}) V(\theta, \dot{\theta})=\left(\frac{1}{\zeta(\theta)}\left[\begin{array}{cc}
-2 \theta-3 & -2 \\
-2 & 2 \theta+3
\end{array}\right]\right)\left[\begin{array}{c}
\frac{\zeta(\theta)}{3} \dot{\theta} \\
0
\end{array}\right] 1
$$

with $\zeta(\theta)=\sqrt{4 \theta^{2}+12 \theta+3}$. Since $S(\theta, \dot{\theta})$ is a symmetric and unitary matrix, $S(\theta, \dot{\theta})^{-1}=S(\theta, \dot{\theta})$ and

$$
S_{2}=\frac{1}{\zeta(\theta)}\left[\begin{array}{ll}
-2 & 2 \theta+3
\end{array}\right]
$$


Furthermore, as $S_{2}(\theta, \dot{\theta}) g(\theta, \dot{\theta}), \quad S_{2}(\theta, \dot{\theta}) b(\theta, \dot{\theta}) \in \mathbb{R}$, they are parallel by definition and the geometric condition (21) is trivially satisfied with

$$
\lambda(\theta, \dot{\theta})=\dot{\theta}+\frac{2}{3} \theta \dot{\theta}-1 .
$$

Finally, using (24) we get

$$
\frac{1}{3} \zeta(\theta) \dot{\theta} \rho^{\prime}=-\frac{4}{3} \zeta(\theta) \dot{\theta} \rho \Longrightarrow \rho(\theta)=\lambda e^{-4 \theta},
$$

thus yielding again the previous results.

In the simulated scenario, we have considered $\theta \in[0,3]$ and $\dot{\theta} \in[-0.5,0.5]$, with a trajectory for $\theta(t)$ as in Fig.1. Let us choose $\lambda=1$, and let us compare the behavior of the closed-loop system obtained with different controllers, designed by applying a slightly modified version of (34), which introduces an additional term $2 \alpha P$ to require the Lyapunov function to decrease with a guaranteed decay rate $\alpha$. In particular, three values for $\alpha$ are considered: $\alpha=0, \alpha=1$ and $\alpha=10$, the first one of which corresponding to the simpler requirement of asymptotic stability. The simulations have been performed starting from the initial state $x(0)=0$. Figs. 2-3 show that the closedloop state trajectory converges to the desired steady-state, with a speed of convergence that depends on the value of $\alpha$ (the bigger $\alpha$ is, the faster the dynamics of the closed-loop system is).

\section{2. $A(\theta, 0)$ not invertible}

In this second example we consider the LPV system described by the plant matrices

$$
A(\theta, \dot{\theta})=\left(\begin{array}{cc}
\theta & 1 \\
\frac{\theta^{2}+\theta-\theta \dot{\theta}-5 \dot{\theta}+\theta^{3}}{\theta^{2}+\theta+1} & \frac{\theta^{2}+\theta+1+\theta \dot{\theta}+2 \dot{\theta}}{\theta^{2}+\theta+1}
\end{array}\right), \quad B(\theta, \dot{\theta})=\left(\begin{array}{l}
1 \\
1
\end{array}\right)
$$

with $\theta \in[-2,-1]$ and $\dot{\theta} \in[-1,1]$.

Since

$$
\operatorname{rank}\{A(\theta, \dot{\theta})\}=\operatorname{rank}\{[A(\theta, \dot{\theta}), b(\theta, \dot{\theta})]\}=1,
$$

the necessary condition for the existence of $v \in \overline{\mathcal{V}}$ is satisfied. By Theorem 1 , any RSP $v(\theta)$ must lie on a linear space of dimension $1+n-\operatorname{rank}\{A(\theta, 0)\}=$ 2 , which in this case coincides with the whole state space. Equation (4) can be used to search for admissible steady-state trajectories for (37). Bearing this in mind, let us seek for RSP of the simple form

$$
v(\theta)=\left(\begin{array}{c}
a \theta+b \\
c \theta+d
\end{array}\right) \text {. }
$$




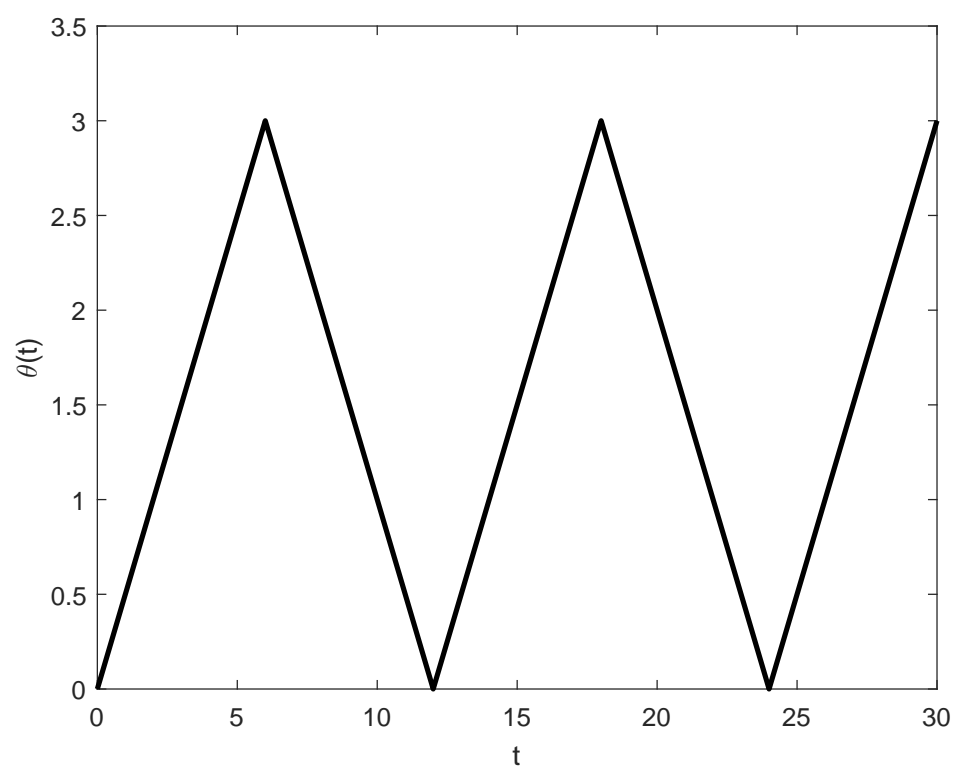

Figure 1: Example 1 - Trajectory of the varying parameter $\theta(t)$.

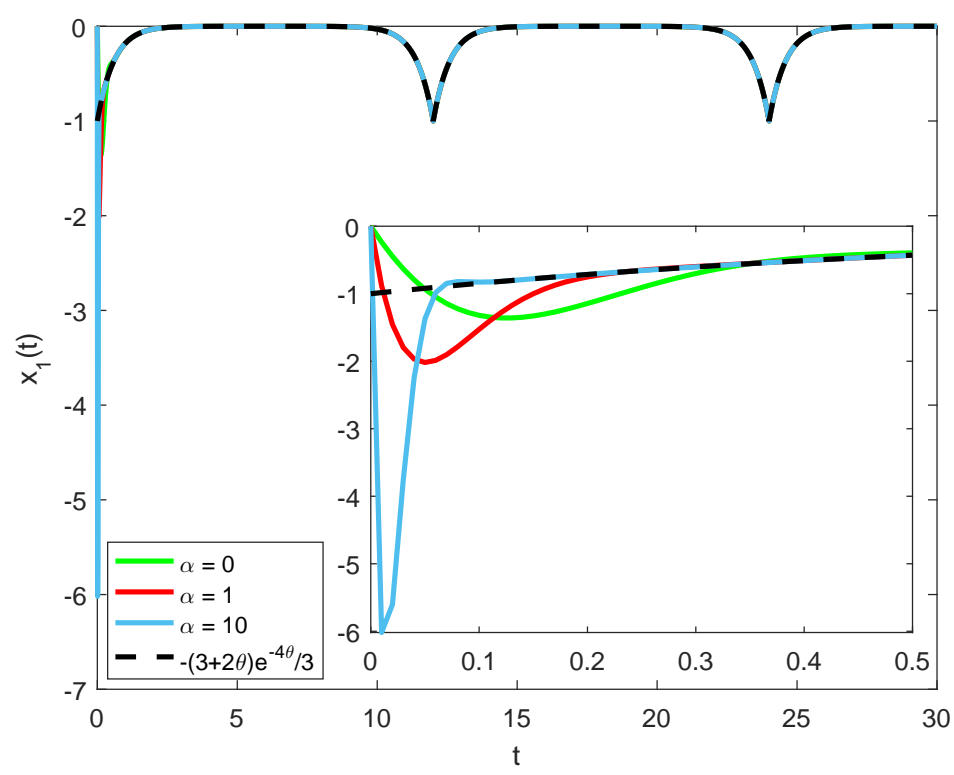

Figure 2: Example 1 - State trajectory $x_{1}(t)$ (closed-loop). 


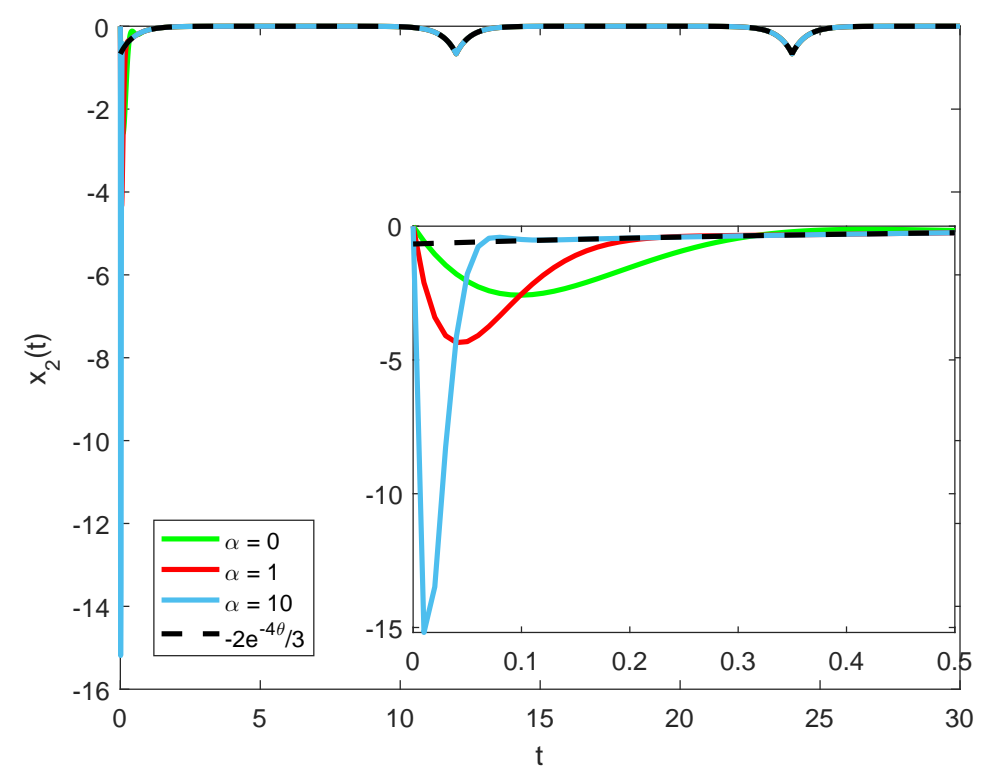

Figure 3: Example 1 - State trajectory $x_{2}(t)$ (closed-loop).

Accordingly, (4) leads to the system

$$
\left\{\begin{array}{l}
a \dot{\theta}=a \theta^{2}+b \theta+c \theta+d+u \\
c \dot{\theta}=\left(\theta-\frac{5+\theta}{\theta^{2}+\theta+1} \dot{\theta}\right)(a \theta+b)+\left(1+\frac{2+\theta}{\theta^{2}+\theta+1} \dot{\theta}\right)(c \theta+d)+u
\end{array}\right.
$$

which admits solution if $c=3 a-b$ and $d=a+2 b$, with input function

$$
u_{v}(\theta, \dot{\theta})=a \dot{\theta}-\left(a \theta^{2}+b \theta+c \theta+d\right) .
$$

In conclusion, as long as the latter arithmetic conditions are fulfilled, the input $u(t)=\lambda u_{v}(\theta, \dot{\theta})$ keeps the LPV system (37) on the steady-state trajectory

$$
x_{\infty}(\theta(t))=\lambda\left(\begin{array}{c}
a \theta+b \\
c \theta+d
\end{array}\right)
$$

Figs. 4-5 show the simulation responses obtained with $\lambda=1, a=2$, $b=1$ and $\theta(t)=-1.5+\sin (t)$. The black dashed line corresponds to the calculated steady-state trajectories, whereas the green solid line corresponds to the simulation with $x_{0}=(-2,-3.5)^{T}$, i.e. with the initial condition on the steady-state. The match between the black and the green lines demonstrates that $(2 \theta(t)+1,5 \theta(t)+4)$ is indeed a steady-state equilibrium for the 
LPV system (37) forced by $u(t)=\lambda u_{v}(\theta, \dot{\theta})$. However, this equilibrium is unstable: in fact a slight variation of the initial condition leads to a divergent behavior, as shown by the red solid lines in Figs. 4-5, which correspond to the simulations obtained with initial condition $x(0)=(-1.99,-3.49)^{T}$.

By using a constant Lyapunov matrix [2] and a gridding approach to reduce the infinite number of design conditions to a finite number [27], a

controller gain $k(\theta, \dot{\theta})$ which stabilizes the LPV system for any $\theta \in[-2,-1]$ and $\dot{\theta} \in[-1,1]$ has been computed. The closed-loop simulation results obtained with an initial condition $x(0)=(3,6.5)^{T}$ (see Figs. 6-7) demonstrates that the input (36) drives asymptotically the LPV system to the chosen steady-state trajectory.

\section{Conclusions and future work}

The characterization of single-input LPV systems admitting scheduled steady-states has been investigated. In particular, steady-state trajectories depending exclusively on the scheduling parameter have been proved to exist and to be reachable if and only if some geometric, structural conditions are met by the plant matrices along with the existence of a solution to a suitable partial differential problem. Such solution can be used to synthesize the control inputs in a closed-form. It is worth to stress that the property of an LPV system to admit a scheduled steady-state is uniform in all possible scheduling parameter trajectories. Furthermore, a Lyapunovbased technique to establish stability or to achieve stabilization of scheduled steady-states has been provided.

Future developments will be focused on a deep analysis of the partial differential problem with the aim of obtaining sharp conditions for the existence of solutions, as well as on the extension of the scheduled state-state concept to multi-input LPV systems. 


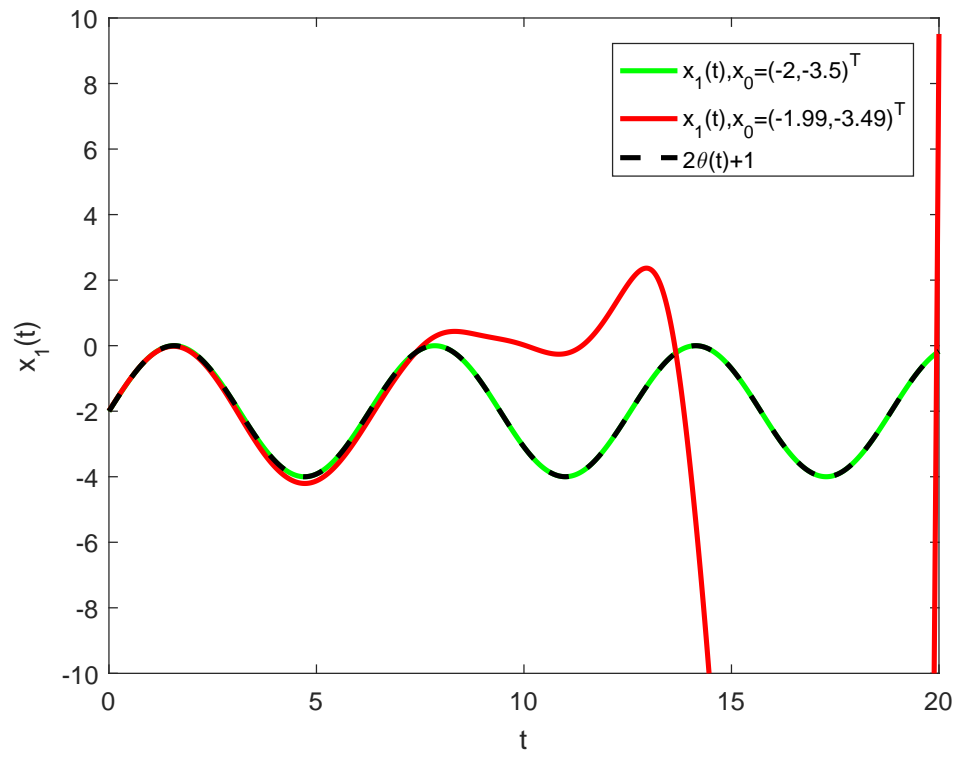

Figure 4: Example 2 - State response $x_{1}(t)$ for $\lambda=1, a=2, b=1$ and $\theta(t)=-1.5+\sin (t)$ (open-loop).

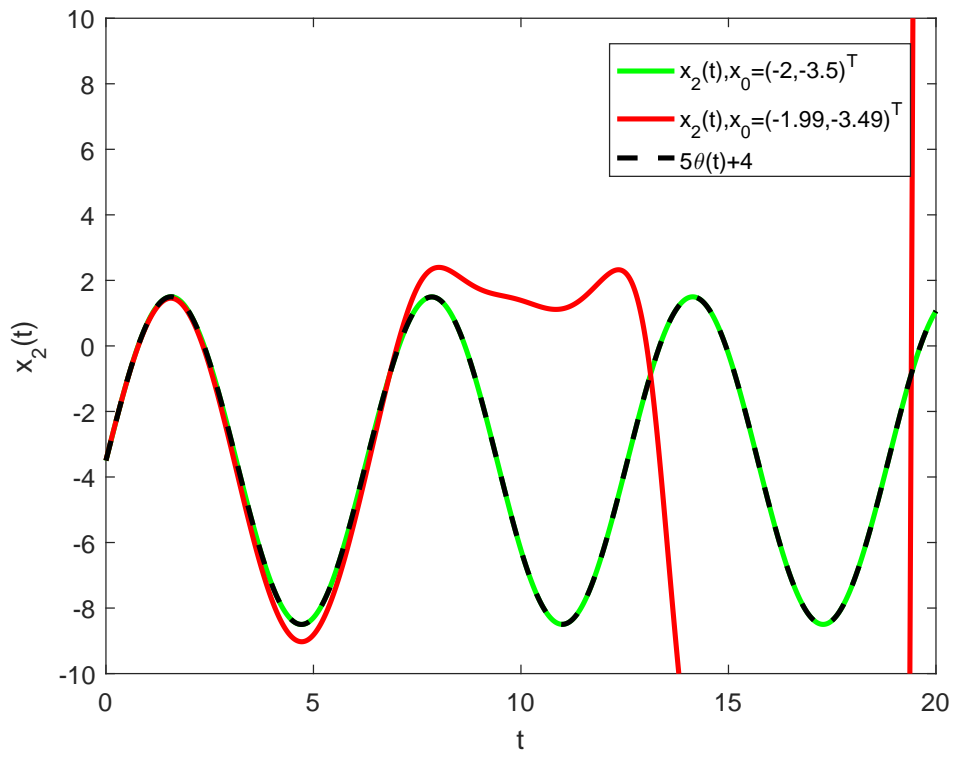

Figure 5: Example 2 - State response $x_{2}(t)$ for $\lambda=1, a=2, b=1$ and $\theta(t)=-1.5+\sin (t)$ (open-loop). 


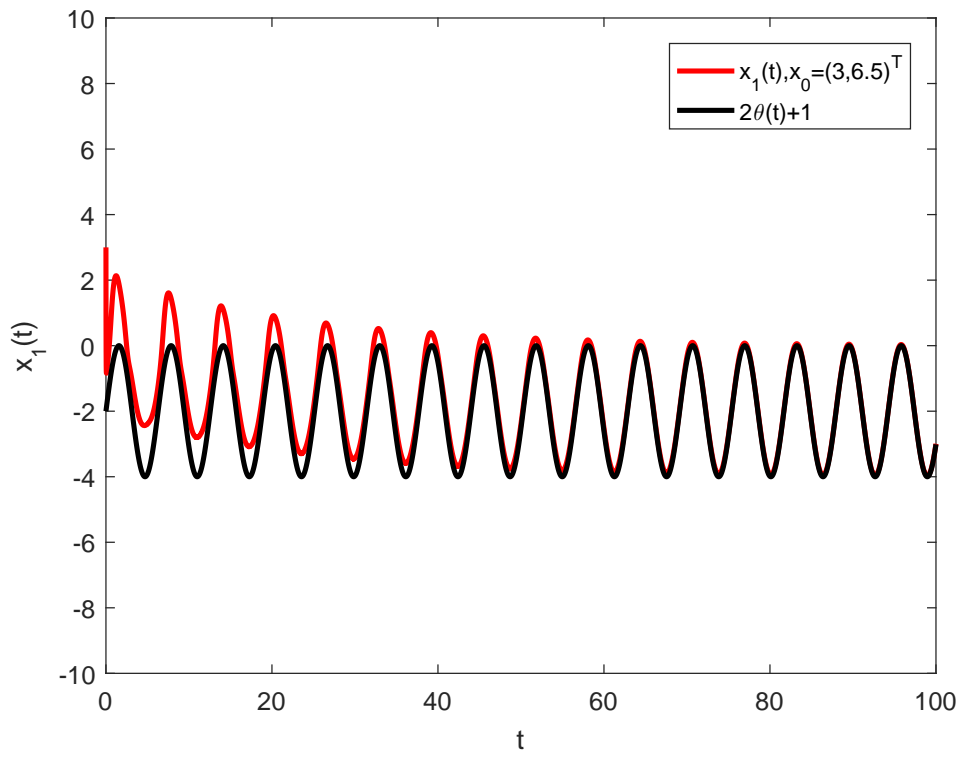

Figure 6: Example 2 - State response $x_{1}(t)$ for $\lambda=1, a=2, b=1$ and $\theta(t)=-1.5+\sin (t)$ (closed-loop).

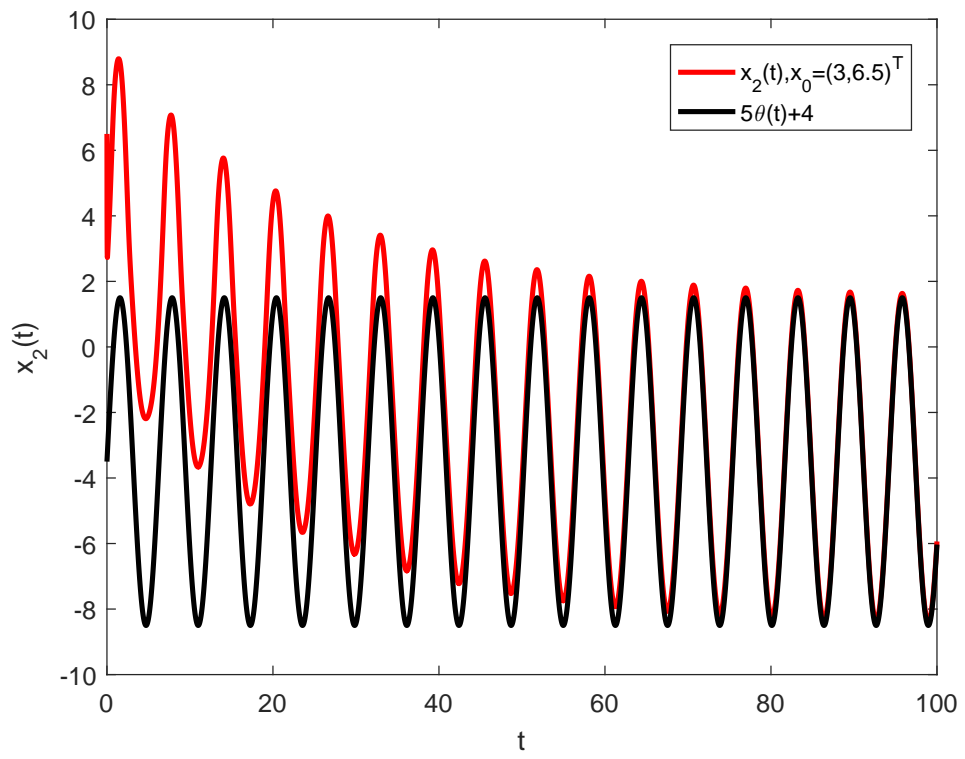

Figure 7: Example 2 - State response $x_{2}(t)$ for $\lambda=1, a=2, b=1$ and $\theta(t)=-1.5+\sin (t)$ (closed-loop). 


\section{Acknowledgements}

This work has been partially funded by AGAUR of the Generalitat de Catalunya through the Advanced Control Systems (SAC) group grant (2017 SGR 482), and by the Spanish State Research Agency through the Maria de Maeztu Seal of Excellence to IRI (MDM-2016-0656) and the grant Juan de la Cierva-Formacion (FJCI-2016-29019).

\section{References}

[1] A. Abdullah and M. Zribi. Model reference control of LPV systems. Journal of the Franklin Institute, 346:854-871, 2009.

[2] Gregory Becker, Andy Packard, Doug Philbrick, and Gary Balas. Control of parametrically-dependent linear systems: A single quadratic lyapunov approach. In American Control Conference, 1993, pages 27952799. IEEE, 1993.

[3] Renato Alves Borges, Vinicius Foletto Montagner, Ricardo CLF Oliveira, Pedro Luis Dias Peres, and P-A Bliman. Parameter-dependent $\mathrm{h} 2$ and $\mathrm{h}$ filter design for linear systems with arbitrarily time-varying parameters in polytopic domains. Signal Processing, 88(7):1801-1816, 2008.

[4] Patricio H Colmegna, Ricardo S Sanchez-Pena, Ravi Gondhalekar, Eyal Dassau, and Frank J Doyle. Switched lpv glucose control in type 1 diabetes. IEEE Transactions on Biomedical Engineering, 63(6):11921200, 2016.

[5] D. Corona, A. Cristofaro, and D. Rotondo. Optimizing output regulation for a class of underactuated lpv systems. In Mediterranean Control Conference, pages 135-140, 2017.

[6] Pepijn B Cox, Siep Weiland, and Roland Toth. Affine parameterdependent lyapunov functions for lpv systems with affine dependence. IEEE Transactions on Automatic Control, 2018, in press.

[7] Gianfranco Gagliardi, Francesco Tedesco, and Alessandro Casavola. A lpv modeling of turbocharged spark-ignition automotive engine oriented to fault detection and isolation purposes. Journal of the Franklin Institute, 2018, in press. 
[8] C. Hoffmann and H. Werner. A survey of linear parameter-varying control applications validated by experiments or high-fidelity simulations. IEEE Transactions on Control Systems Technology, 23(2):416$433,2015$.

[9] Alberto Isidori. Nonlinear control systems. Springer Science \& Business Media, 2013.

[10] Mohammad Bagher Abolhasani Jabali and Mohammad Hosein Kazemi. Uncertain polytopic lpv modelling of robot manipulators and trajectory tracking. International Journal of Control, Automation and Systems, 15(2):883-891, 2017.

[11] Sung Hyun Kim. H2 control of markovian jump lpv systems with measurement noises: Application to a dc-motor device with voltage fluctuations. Journal of the Franklin Institute, 354(4):1784-1800, 2017.

[12] A. Kwiatkowski, M.-T. Boll, and H. Werner. Automated Generation and Assessment of Affine LPV Models. In Proceedings of the 45th IEEE Conf. on Decision and Control, pages 6690-6695, 2006.

[13] Manas Mejari, Dario Piga, and Alberto Bemporad. A bias-correction method for closed-loop identification of linear parameter-varying systems. Automatica, 87:128-141, 2018.

[14] M. Q. Nguyen, J. M. Gomes da Silva Jr., O. Sename, and L. Dugard. A state feedback input constrained control design for a 4-semi-active damper suspension system: a quasi-LPV approach. In Proceedings of the 8th IFAC Symposium on Robust Control Design (ROCOND), pages 259-264, 2015.

[15] Katsuhiko Ogata and Yanjuan Yang. Modern control engineering, volume 4. Prentice hall India, 2002.

[16] Hangli Ren and Guangdeng Zong. Asynchronous input-output finitetime filtering for switched lpv systems. Journal of the Franklin Institute, 354(14):6292-6311, 2017.

[17] Mickael Rodrigues, Habib Hamdi, Naceur Benhadj Braiek, and Didier Theilliol. Observer-based fault tolerant control design for a class of lpv descriptor systems. Journal of the Franklin Institute, 351(6):3104-3125, 2014. 
[18] D. Rotondo, V. Puig, F. Nejjari, and J. Romera. A fault-hiding approach for the switching quasi-LPV fault-tolerant control of a fourwheeled omnidirectional mobile robot. IEEE Transactions on Industrial Electronics, 62(6):3932-3944, 2015.

[19] Damiano Rotondo. Advances in gain-scheduling and fault tolerant control techniques. Springer, 2017.

[20] Damiano Rotondo, Andrea Cristofaro, Tor Arne Johansen, F Nejjari, and V Puig. State estimation and decoupling of unknown inputs in uncertain lpv systems using interval observers. International Journal of Control, 91(8):1944-1961, 2018.

[21] Damiano Rotondo, Andrea Cristofaro, Tor Arne Johansen, Fatiha Nejjari, and Vicenç Puig. Diagnosis of icing and actuator faults in uavs using lpv unknown input observers. Journal of Intelligent $\&$ Robotic Systems, pages 1-15, 2017.

[22] Damiano Rotondo, Fatiha Nejjari, and Vicenç Puig. A virtual actuator and sensor approach for fault tolerant control of lpv systems. Journal of Process Control, 24(3):203-222, 2014.

[23] Damiano Rotondo, Vicenc Puig, Fatiha Nejjari, and Marcin Witczak. Automated generation and comparison of takagi-sugeno and polytopic quasi-lpv models. Fuzzy Sets and Systems, 277:44-64, 2015.

[24] Masayuki Sato. Gain-scheduled flight controller using bounded inexact scheduling parameters. IEEE Transactions on Control Systems Technology, 26(3):1074-1082, 2018.

[25] Olivier Sename, Peter Gaspar, and József Bokor. Robust control and linear parameter varying approaches: application to vehicle dynamics, volume 437. Springer, 2013.

[26] J. S. Shamma. An overview of LPV systems. In J. Mohammadpour and C. Scherer, editors, Control of Linear Parameter Varying Systems with Applications. Springer, 2012.

[27] Xiukun Wei and Luigi del Re. Gain scheduled $h_{\infty}$ control for air path systems of diesel engines using lpv techniques. IEEE transactions on control systems technology, 15(3):406-415, 2007. 
[28] Zhi-Hui Zhang and Guang-Hong Yang. Fault detection for discretetime uncertain lpv systems using non-minimal order filter. Journal of the Franklin Institute, 355(2):902-921, 2018.

[29] Kongwei Zhu, Dan Ma, and Jun Zhao. Event triggered control for a switched lpv system with applications to aircraft engines. IET Control Theory \& Applications, 12(10):1505-1514, 2018. 